IJMMS 28:11 (2001) 653-661

PII. S0161171201007748

http://ijmms.hindawi.com

(C) Hindawi Publishing Corp.

\title{
STRESS WAVES IN A MICROPERIODIC LAYERED ELASTIC SOLID REVISITED
}

\author{
JÓZEF IGNACZAK and ELŻBIETA MRÓWKA-MATEJEWSKA
}

(Received 10 June 2001)

\begin{abstract}
A one-dimensional pure stress initial boundary value problem of linear elastodynamics for a microperiodic layered semi-space in which a microstructural length is taken into account is revisited. Also, the plane stress harmonic waves propagating in a microperiodic layered infinite elastic space are discussed. It is shown that (i) for a particular system of the length and time units the transient stress waves in the microperiodic layered semi-space are independent of the microstructural length, and (ii) there are two dispersive plane stress harmonic waves propagating in a microperiodic layered infinite elastic space. The graphs illustrating the transient stress waves in the semi-space and the dispersion of harmonic waves in the infinite space are included.
\end{abstract}

2000 Mathematics Subject Classification. 74H05, 74H25, 74H45, 74J05, 74Q10.

1. Basic field equations for a microperiodic layered elastic semi-space. Consider a layered semi-infinite elastic solid composed of an infinite number of identical subunits that are mechanically bonded to form a spatially periodic pattern as shown in Figure 1.1. Each subunit consists of two layers that, in general, have different dimensions and are made of different homogeneous isotropic elastic materials. Let $l_{i}, \rho_{i}, \lambda_{i}$, and $\mu_{i}(i=1,2)$, respectively, denote the physical dimension, density, Lamé modulus, and shear modulus of the $i$ th layer in a subunit. If the interface conditions between any two adjacent layers are assumed to be of an ideal mechanical contact type, that is, the displacement and stress vectors are continuous across an interface, and a mechanical load is uniformly distributed over the boundary $x=0$ for every time $t \geq 0$, an elastic process in the layered semi-space can be described by a solution to a one-dimensional initial boundary value problem of classical elastodynamics. In such a problem the field equations of homogeneous isotropic elastodynamics are to be satisfied for each layer and suitable initial, interface, and boundary conditions at $x=0$ and $x=\infty$ are to be met. Since an exact solution to the problem is not feasible, the classical formulation for the layered semi-space is replaced by the approximate one of a refined average theory (RAT), (see $[1,2,4]$ ). The field equations of the approximate theory read: the $h$-approximation of the displacement field

$$
u(x, t)=U(x, t)+h(x) V(x, t) .
$$

The equations of motion

$$
S_{x}-\langle\rho\rangle U_{t t}=0, \quad H+\left\langle\rho h^{2}\right\rangle V_{t t}=0 .
$$




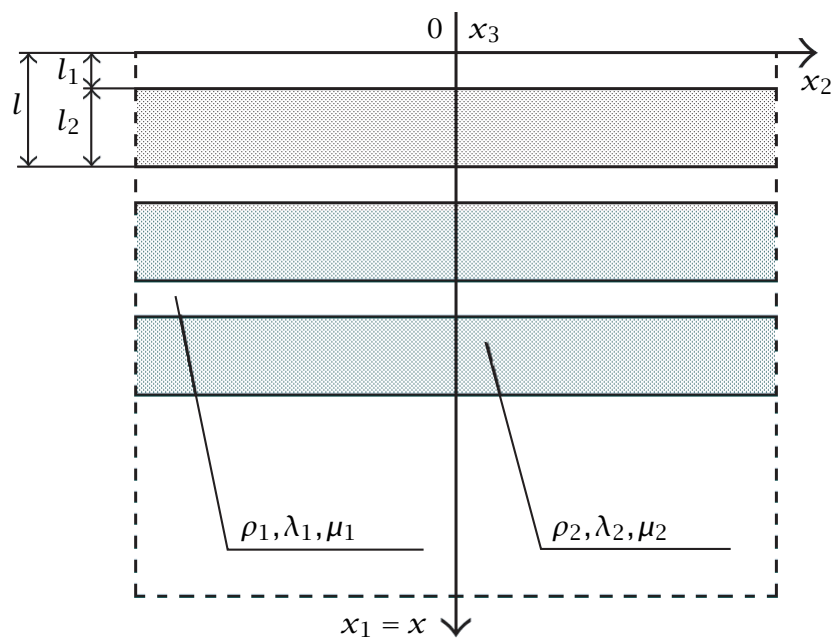

FIGURE 1.1. Configuration of a microperiodic layered elastic semi-space.

The constitutive relations

$$
\begin{aligned}
& S=\langle\Lambda\rangle U_{x}+\left\langle\Lambda h_{x}\right\rangle V, \\
& H=\left\langle\Lambda h_{x}\right\rangle U_{x}+\left\langle\Lambda h_{x}^{2}\right\rangle V .
\end{aligned}
$$

Here, $h=h(x)$ is a dimensionless oscillating periodic function on $[0, \infty)$ with period $l$ that satisfies the conditions

$$
\langle h\rangle=0, \quad\langle\eta h\rangle=0, \quad\left\langle\eta h_{x}\right\rangle \neq 0,
$$

for any function $\eta=\eta(x)$ on $[0, l]$ of the form

$$
\eta(x)= \begin{cases}\eta_{1} & \text { for } 0 \leq x<l_{1}, \\ \eta_{2} & \text { for } l_{1} \leq x \leq l,\end{cases}
$$

where $\eta_{1}$ and $\eta_{2}$ are constants $\left(\eta_{1} \neq \eta_{2}\right)$; and for any function $F=F(x)$ on $[0, l]$ the symbol $\langle\cdot\rangle$ represents the mean value of $F$ on $[0, l]$

$$
\langle F\rangle=\frac{1}{l} \int_{0}^{l} F(x) d x .
$$

In addition, the function $h=h(x)$ satisfies the asymptotic estimate

$$
h(x)=l 0(1) \quad \text { as } l \longrightarrow 0 .
$$

If $l$ is small, the function $h=h(x)$ represents a micro-periodic shape function. A typical micro-periodic shape function $h=h(x)$ restricted to the interval $[0, l]$ is shown in Figure 1.2.

Other symbols in (1.1), (1.2), and (1.3) have the following meaning. In (1.1) the function $u$ represents a displacement in the $x$-direction; $U$ is a macro-displacement in 


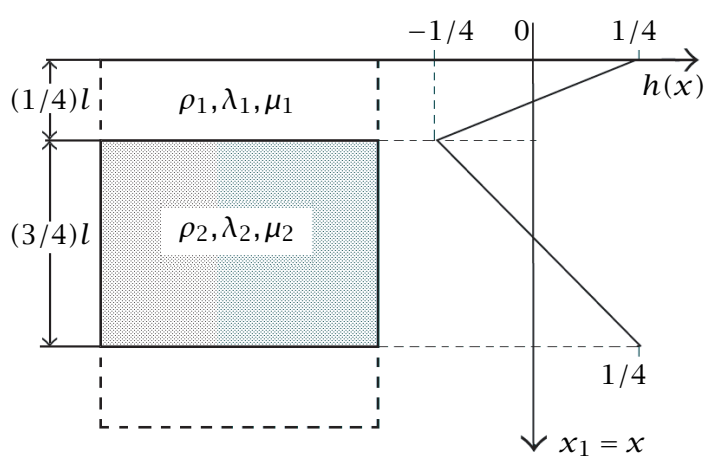

FIGURE 1.2. A microperiodic shape function $h=h(x)$ over the interval $0 \leq$ $x \leq l$.

the $x$-direction and $V$ is a displacement corrector. In (1.2) and (1.3) the function $S$ is a stress component in the $x$-direction and $H$ is a body force component in the $x$ direction; moreover, $\Lambda=\lambda+2 \mu$, where $\lambda$ and $\mu$ are Lamé moduli, and $\rho$ is the density. The subscripts in (1.2) and (1.3) indicate partial derivatives.

The kinetic energy density $K=K(x, t)$ and the potential energy density $P=P(x, t)$, associated with (1.2) and (1.3) are represented by the functions

$$
\begin{aligned}
& K(x, t)=\frac{1}{2}\left[\langle\rho\rangle U_{t}^{2}+\left\langle\rho h^{2}\right\rangle V_{t}^{2}\right], \\
& P(x, t)=\frac{1}{2}\left[\langle\Lambda\rangle U_{x}^{2}+2\left\langle\Lambda h_{x}\right\rangle U_{x} V+\left\langle\Lambda h_{x}^{2}\right\rangle V^{2}\right],
\end{aligned}
$$

respectively.

Note that (1.2) and (1.3) form a complete set of four field equations of the onedimensional theory for the four unknowns $U, V ; S$, and $H$. By eliminating the functions $U, V$, and $H$ from (1.2) and (1.3) we arrive at the stress equation for $S$

$$
\left(\frac{\partial^{2}}{\partial x^{2}}-\frac{1}{c^{2}} \frac{\partial^{2}}{\partial t^{2}}\right)\left(\frac{\partial^{2}}{\partial t^{2}}+\kappa^{2}\right) S-\frac{\omega^{2}}{c^{2}} \frac{\partial^{2}}{\partial t^{2}} S=0
$$

where

$$
\begin{gathered}
c=\frac{\langle\Lambda\rangle^{1 / 2}}{\langle\rho\rangle^{1 / 2}}, \quad \kappa=\Omega \frac{\left\langle\Lambda^{*}\right\rangle^{1 / 2}}{\langle\Lambda\rangle^{1 / 2}}, \\
\omega=\Omega\left(1-\frac{\left\langle\Lambda^{*}\right\rangle}{\langle\Lambda\rangle}\right)^{1 / 2}, \quad \Omega=\frac{\left\langle\Lambda h_{x}^{2}\right\rangle^{1 / 2}}{\left\langle\rho h^{2}\right\rangle^{1 / 2}}, \\
\left\langle\Lambda^{*}\right\rangle=\langle\Lambda\rangle-\frac{\left\langle\Lambda h_{x}\right\rangle^{2}}{\left\langle\Lambda h_{x}^{2}\right\rangle} .
\end{gathered}
$$

Clearly, $c$ has the dimension of velocity, and $\Omega$ has the dimension of frequency, that is,

$$
[c]=\left[L T^{-1}\right], \quad[\Omega]=[\kappa]=[\omega]=\left[T^{-1}\right],
$$

where $L$ and $T$ stand for the length and time units, respectively; and [ $\cdot]$ represents the dimension of a physical quantity. 
As a result, for the microperiodic layered semi-space subject to homogeneous initial conditions and a uniform pressure $s=s(t)$ on its boundary $x=0$, the following pure stress initial boundary value problem can be formulated.

Find a stress field $S=S(x, t)$ on $[0, \infty) \times[0, \infty)$ that satisfies the field equation

$$
\left(\frac{\partial^{2}}{\partial x^{2}}-\frac{1}{c^{2}} \frac{\partial^{2}}{\partial t^{2}}\right)\left(\frac{\partial^{2}}{\partial t^{2}}+\kappa^{2}\right) S-\frac{\omega^{2}}{c^{2}} \frac{\partial^{2}}{\partial t^{2}} S=0 \text { for } x>0, t>0
$$

subject to the initial conditions

$$
S(x, 0)=0, \quad \frac{\partial}{\partial t} S(x, 0)=0, \quad \frac{\partial^{2}}{\partial t^{2}} S(x, 0)=0, \quad \frac{\partial^{3}}{\partial t^{3}} S(x, 0)=0, \quad \text { for } x>0
$$

and the boundary condition

$$
S(0, t)=-s(t) \text { for } t>0,
$$

where $s=s(t)$ is a prescribed function. Moreover, the function $S$ and its partial derivatives of a finite order are to vanish as $x \rightarrow \infty$ for every $t>0$.

If a solution $S$ to the problem (1.13), (1.14), and (1.15) is found, the functions $H, U$, and $V$ are computed from the formulas

$$
\begin{aligned}
& H(x, t)=\frac{\left\langle\Lambda h_{x}\right\rangle}{\langle\Lambda\rangle} \int_{0}^{t} \cos \kappa(t-\tau) \frac{\partial S}{\partial \tau}(x, \tau) d \tau \\
& U(x, t)=\frac{1}{\langle\rho\rangle} \int_{0}^{t}(t-\tau) \frac{\partial S}{\partial x}(x, \tau) d \tau \\
& V(x, t)=-\frac{1}{\left\langle\rho h^{2}\right\rangle} \int_{0}^{t}(t-\tau) H(x, \tau) d \tau .
\end{aligned}
$$

Also, note that the pure stress problem (1.13), (1.14), and (1.15) contains two high frequency parameters $\kappa$ and $\omega$, since, by (1.7) and (1.10), we have

$$
\kappa=l^{-1} 0(1), \quad \omega=l^{-1} 0(1), \quad \text { as } l \longrightarrow 0 .
$$

\section{Uniqueness and Green's function theorem for the pure stress problem}

UNIQUENESS THEOREM. The pure stress initial boundary value problem (1.13), (1.14), and (1.15) may have at most one solution.

Proof. See [2, Theorem 3.1].

REPRESENTATION THEOREM. A solution to problem (1.13), (1.14), and (1.15) admits the representation

$$
S(x, t)=\frac{1}{\kappa} \int_{0}^{t} \sin \kappa(t-\tau) \Sigma(x, \tau) d \tau,
$$

where

$$
\Sigma(x, t)=\int_{0}^{t} G(x, t-\tau)\left[\ddot{s}(\tau)+\kappa^{2} s(\tau)\right] d \tau
$$


and $G=G(x, t)$ is a Green's function for the integro-differential problem. Find a function $G=G(x, t)$ that satisfies the equation

$$
\left(\frac{\partial^{2}}{\partial x^{2}}-\frac{1}{c^{2}} \frac{\partial^{2}}{\partial t^{2}}\right) G-\frac{\omega^{2}}{c^{2}} \int_{0}^{t} \cos \kappa(t-\tau) \frac{\partial G}{\partial \tau}(x, \tau) d \tau=0 \quad \text { for } x \geq 0, t \geq 0
$$

the initial conditions

$$
G(x, 0)=0, \quad \frac{\partial}{\partial t} G(x, 0)=0, \quad \text { for } x \geq 0,
$$

the boundary condition

$$
G(0, t)=-\delta(t),
$$

and vanishing conditions at infinity. In (2.5) $\delta=\delta(t)$ is the Dirac delta function.

Proof. See [2, equations (38)-(42)].

GREEN'S FUNCTION THEOREM. (i) A solution to the integro-differential problem (2.3), (2.4), and (2.5) admits the series representation of the Neumann's type

$$
G(x, t)=-\delta\left(t-\frac{x}{c}\right)-H\left(t-\frac{x}{c}\right) g\left(x, t-\frac{x}{c}\right),
$$

where $H=H(t)$ is the Heaviside function

$$
\begin{gathered}
H(t)= \begin{cases}1 & \text { for } t>0, \\
0 & \text { for } t<0,\end{cases} \\
g(x, t)=\sum_{n=1}^{\infty} \frac{(-1)^{n}}{n !}\left(\frac{x \omega^{2}}{2 c}\right)^{n}\left[\{\cos \kappa t\}^{n}+n \sum_{m=1}^{\infty} \frac{(-1)^{m}}{m !}\left(\frac{\omega^{2}}{4}\right)^{m}\right. \\
\left.\times \frac{(n+2 m-1) !}{(n+m) !(m-1) !}\left\{t^{m-1}\right\}\{\cos \kappa t\}^{n+m}\right] \quad \text { for } x \geq 0, t \geq 0 .
\end{gathered}
$$

Here, for arbitrary functions $a=a(t)$ and $b=b(t)$ on $[0, \infty)$, the symbol $\{a\}\{b\}$ represents the convolution product of $a=a(t)$ and $b=b(t)$ defined by (see [3])

$$
\{a\}\{b\}=\int_{0}^{t} a(t-\tau) b(\tau) d \tau \equiv a * b .
$$

In particular, $\{f\}^{n}$ represents the $n$th convolutional power of a function $\{f(t)\} \equiv f(t)$.

(ii) The series representation of $g=g(x, t)$ and its partial derivatives of a finite order converge uniformly for every point $(x, t) \in(0, \infty) \times(0, \infty)$ and for arbitrary finite parameters $\omega, \kappa$, and $c$.

Proof. See [2, equations (43)-(47)].

It follows from the representation theorem that if $s(t)=\delta(t)$ then $S(x, t) \equiv G(x, t)$. Hence, the Green's function for the pure stress problem represents a transient stress wave in the microperiodic layered elastic semi-space $x>0$ subject to a unit impulsive pressure on its boundary $x=0$. 
Also, it follows from (2.6) and (2.7) that the transient stress wave described by the function $G=G(x, t)$ depends strongly on the layering period $l$ through the frequency parameters $\omega$ and $\kappa$ which become large as $l \rightarrow 0$ (see (1.17)). In the following we prove that the pure stress problem (1.13), (1.14), and (1.15) can be reduced to a dimensionless form in which the microstructural length is absent. More precisely, we prove the following theorem.

THE SCALE-INDEPENDENT STRESS WAVE THEOREM. For a particular system of the length and time units, RAT description of the stress waves in a microperiodic layered elastic semi-space is independent of the layering period.

Proof. Let $l_{0}$ and $t_{0}$ denote the length and time units, respectively, defined by

$$
l_{0}=\frac{\langle\Lambda\rangle^{1 / 2}}{\langle\rho\rangle^{1 / 2}} \frac{1}{\omega}, \quad t_{0}=\frac{1}{\omega},
$$

and let $\xi$ and $\tau$ denote the dimensionless space and time variables, respectively. Then the governing integro-differential equation (2.3) reduces to the dimensionless form

$$
\left(\frac{\partial^{2}}{\partial \xi^{2}}-\frac{\partial^{2}}{\partial \tau^{2}}\right) \hat{G}(\xi, \tau)-\int_{0}^{\tau} \cos \hat{\kappa}(\tau-u) \frac{\partial \hat{G}}{\partial u}(\xi, u) d u=0
$$

where

$$
\hat{\kappa}=\frac{\left\langle\Lambda^{*}\right\rangle^{1 / 2}}{\left[\langle\Lambda\rangle-\left\langle\Lambda^{*}\right\rangle\right]^{1 / 2}}
$$

and a stress wave in the semi-space $\xi \geq 0$ for every $\tau \geq 0$ is represented by the $l$ independent formula

$$
\hat{G}(\xi, \tau)=-\delta(\tau-\xi)-H(\tau-\xi) \hat{g}(\xi, \tau-\xi),
$$

where

$$
\begin{aligned}
\hat{g}(\xi, \tau)= & \sum_{n=1}^{\infty} \frac{(-1)^{n}}{n !} \frac{\xi^{n}}{2^{n}}\{\cos \hat{\kappa} \tau\}^{n} \\
& +\sum_{n=1}^{\infty} \frac{(-1)^{n}}{(n-1) !} \frac{\xi^{n}}{2^{n}} \sum_{m=1}^{\infty} \frac{(-1)^{m}(n+2 m-1) !}{m !(n+m) !(m-1) !} \frac{1}{4^{m}}\left\{\tau^{m-1}\right\}\{\cos \hat{\kappa} \tau\}^{n+m}
\end{aligned}
$$

This completes the proof of the theorem.

Note that the functions $\hat{G}$ and $\hat{g}$ in (2.10), (2.11), (2.12), and (2.13) are dimensionless. In particular, $\hat{G}=\hat{G}(\xi, \tau)$ represents the dimensionless stress related to the nominal stress $S(x, t)=G(x, t)$ through the formula

$$
\hat{G}=t_{0} G \text {. }
$$

The dimensionless material parameter $\hat{\kappa}$ ranges over the semi-infinite interval

$$
0<\hat{\kappa}<\infty .
$$

The stress $\hat{S}=\hat{G}$ as a function of $\tau$ at the cross-section $\xi=1$ for $\hat{\kappa}=1.5$ and $\hat{\kappa}=2.0$ are shown in Figures 2.1 and 2.2, respectively. A part of $\hat{S}$ represented by the Dirac delta function $-\delta(\tau-1)$ is not shown in these figures. 


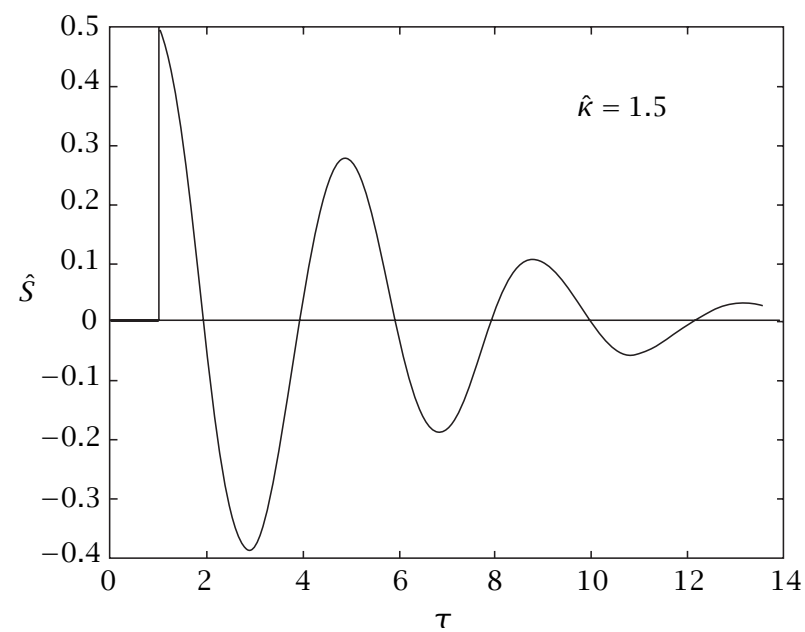

FIGURE 2.1. The stress $\hat{S}$ as a function of dimensionless time $\tau(0 \leq \tau \leq 14)$ at the cross-section $\xi=1$ and for $\hat{\kappa}=1.5$.

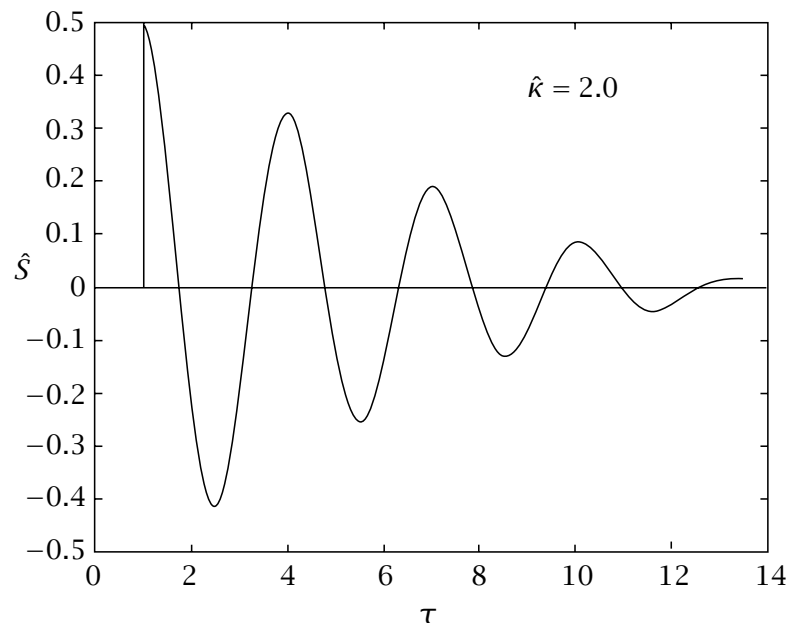

FIGURE 2.2. The stress $\hat{S}$ as a function of dimensionless time $\tau(0 \leq \tau \leq 14)$ at the cross-section $\xi=1$ and for $\hat{\kappa}=2.0$.

3. Dispersion of the harmonic stress waves in a microperiodic layered infinite elastic space

THE DISPERSION THEOREM. There are two plane stress waves of the form

$$
\hat{S}=\hat{S}(\xi, \tau)=S_{0} \exp [i(k \xi-\omega \tau)]
$$




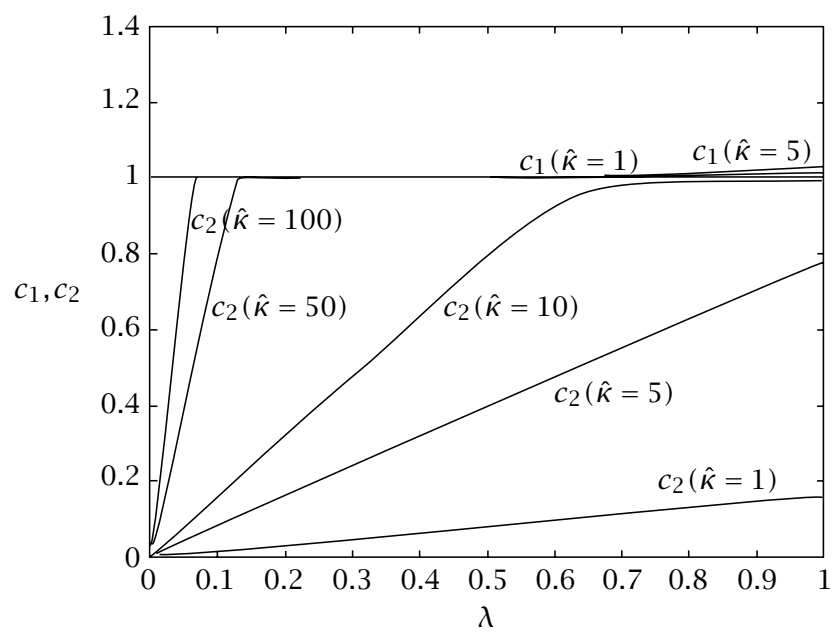

Figure 3.1. The velocities $c_{1}$ and $c_{2}$ as functions of $\lambda(0 \leq \lambda \leq 1)$ for fixed values of $\hat{\kappa}$.

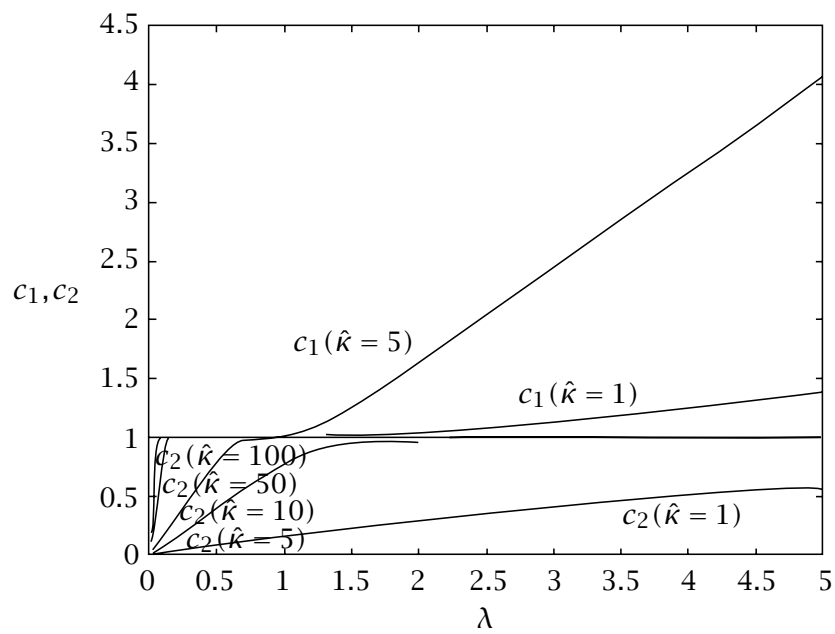

FIGURE 3.2. The velocities $c_{1}$ and $c_{2}$ as functions of $\lambda(0 \leq \lambda \leq 5)$ for fixed values of $\hat{\kappa}$.

where $k=$ wave number, $\omega=$ frequency, $c=\omega / k=$ velocity, propagating in a microperiodic layered infinite elastic body with the velocities

$$
c_{1.2}(\lambda ; \hat{\kappa})=\frac{1}{\sqrt{2}}\left\{\left(1+\frac{1+\hat{\kappa}^{2}}{4 \pi^{2}} \lambda^{2}\right) \pm\left[\left(1+\frac{1+\hat{\kappa}^{2}}{4 \pi^{2}} \lambda^{2}\right)^{2}-\frac{\hat{\kappa}^{2} \lambda^{2}}{\pi^{2}}\right]^{1 / 2}\right\}^{1 / 2},
$$

where $\lambda=2 \pi / k$ is the length of a wave, $\left(S_{0}>0\right)$. Hence, both these waves are dispersive. 
Proof. The proof of this theorem is based on the observation that a plane harmonic wave satisfies the governing equation (see (1.9) reduced to the dimensionless form)

$$
\left(\frac{\partial^{2}}{\partial \xi^{2}}-\frac{\partial^{2}}{\partial \tau^{2}}\right)\left(\frac{\partial^{2}}{\partial \tau^{2}}+\hat{\kappa}^{2}\right) \hat{S}-\frac{\partial^{2}}{\partial \tau^{2}} \hat{S}=0 \text { for }|\xi|<\infty, \tau>0
$$

By substituting $\hat{S}$ from (3.1) into (3.3) and getting rid of the exponential factor we obtain an algebraic equation for $c$. It follows then that there are only two physically admissible velocities $c_{1}$ and $c_{2}$ that satisfy the algebraic equation, and these velocities are given by (3.2). This completes the proof of the theorem.

The velocities $c_{1}$ and $c_{2}$ as functions of $\lambda$ for fixed values of $\hat{\kappa}$ are shown in Figures 3.1 and 3.2. It follows from these figures that a harmonic stress wave propagating with the velocity $c_{1}$ is almost dispersionless over a range of small wave lengths, while that propagating with the velocity $c_{2}$ is almost dispersionless for large $\hat{\kappa}$ and $\lambda$. Otherwise, both these waves are strongly dispersive.

\section{REFERENCES}

[1] J. Ignaczak, Elastodynamics of a microperiodic layered semi-space, Continuous Models of Mechanical Phenomena in Material Systems and in Environment, Polytechnic Press, Poznań, 1999, pp. 25-56 (Polish).

[2] _ Stress waves in a microperiodic layered elastic semi-space, Proceedings of the 15th Brazilian Congress of Mechanical Engineering, COBEM99, November 22-26, 1999, Aquas de Lindoia, Sao Paulo, Brazil, 1999, pp. 1-10.

[3] J. Mikusiński, Operational Calculus, International Series of Monographs on Pure and Applied Mathematics, vol. 8, Pergamon Press, New York, 1959. MR 21\#4333. Zbl 0088.33002.

[4] C. Woźniak and M. Woźniak, Modelling in Dynamics of Composites, IFTR, Report No 25, Polish Academy of Sciences, Warsaw, 1995 (Polish).

Józef Ignaczak: Center of Mechanics, Institute of Fundamental Technological Research, Polish ACAdemy of Sciences, ŚWi ETOKRZySKa 21, 00-049 WARSAW, Poland

E-mail address: jignacz@ippt.gov.p1

Elżbieta Mrówka-Matejewska: Center of Mechanics, Institute of Fundamental TeChNological ReSEarch, Polish ACAdemy of Sciences, Świ ETOKRZYSKa 21, 00-049 WaRSAW, POLAND 


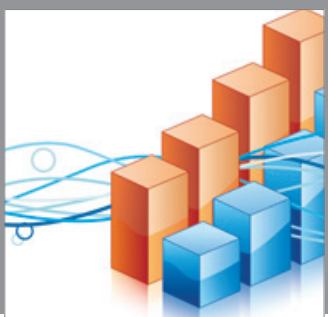

Advances in

Operations Research

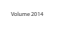

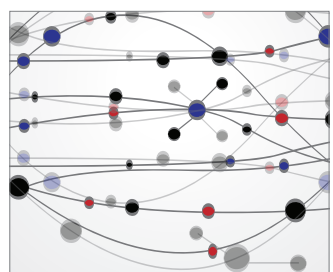

\section{The Scientific} World Journal
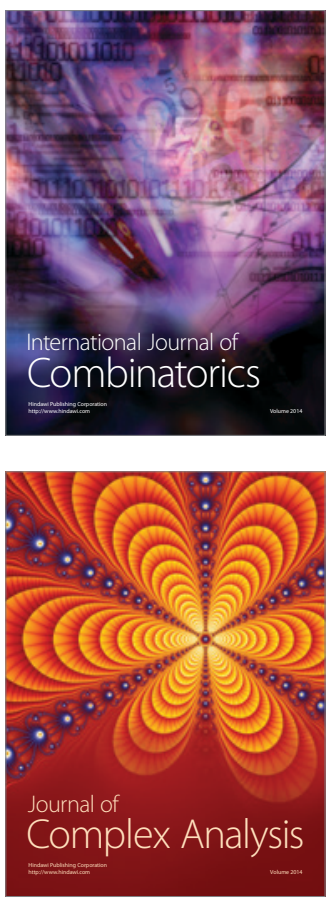

International Journal of

Mathematics and

Mathematical

Sciences
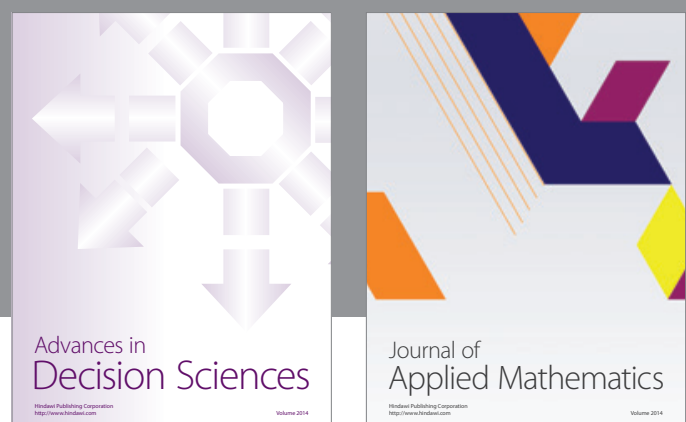

Journal of

Applied Mathematics
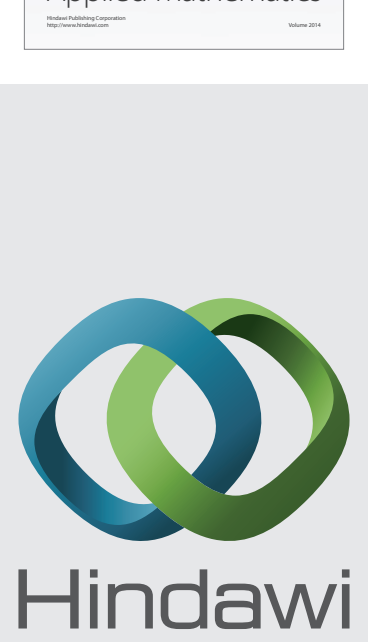

Submit your manuscripts at http://www.hindawi.com
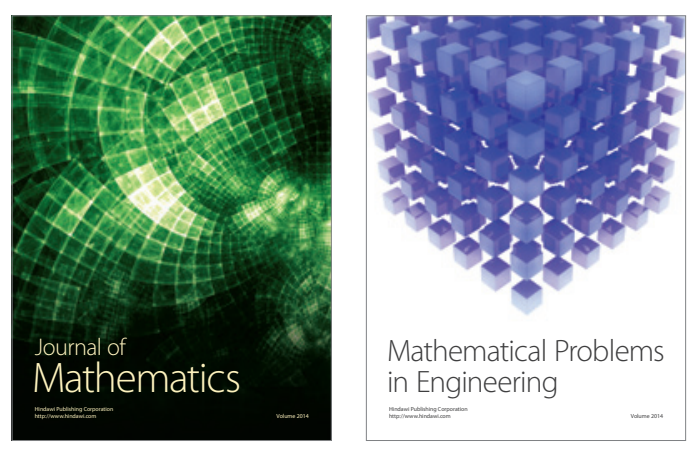

Mathematical Problems in Engineering
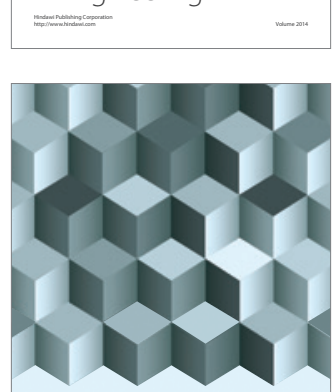

Journal of

Function Spaces
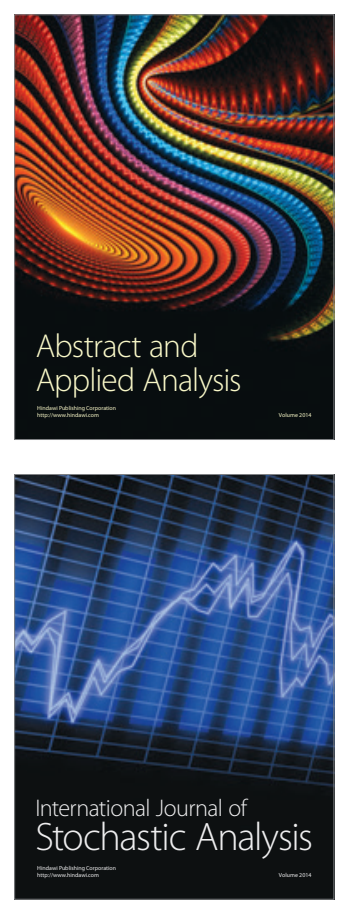

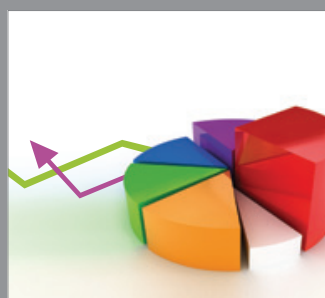

ournal of

Probability and Statistics

Promensencen
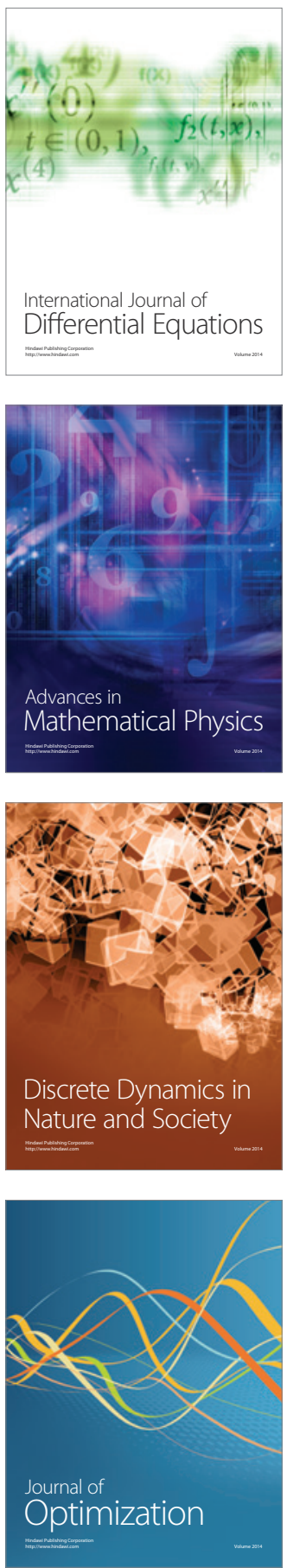